\title{
Prepregnancy Overweight and Obesity Are Associated with an Increased Risk of Preterm Birth in Chinese Women
}

\author{
Xiu Juan Su ${ }^{a}$ Shi Jia Huang ${ }^{b} \quad$ Xiang Li $^{b} \quad$ Qiao Ling Du ${ }^{b}$ \\ ${ }^{a}$ Clinical Research Center, Shanghai First Maternity and Infant Hospital, Tongji University \\ School of Medicine, Shanghai, China; ${ }^{b}$ Department of Obstetrics, Shanghai First Maternity \\ and Infant Hospital, Tongji University School of Medicine, Shanghai, China
}

\section{Keywords}

Body mass index $\cdot$ Maternal obesity $\cdot$ Obesity $\cdot$ Overweight $\cdot$ Preterm birth

\begin{abstract}
Introduction: The association between obesity before pregnancy and preterm birth varies with age and ethnicity. Objective: To study the association between early body mass index (BMI) and risk of preterm birth in Chinese women. Methods: This was a hospital-based retrospective cohort study including 36,596 Chinese women who gave birth to a live singleton infant from 2015 to 2018. Women were classified as underweight (BMI <18.5), normal weight (BMI 18.5 to $<23$ ), overweight (BMI 23 to $<27.5$ ), or obese (BMI $\geq 27.5$ ) according to the most recent criteria for Asian women. Multivariate log-binomial regression models were used to estimate the relative risks (RRs) and 95\% confidence intervals $(95 \% \mathrm{Cls})$ for preterm birth among different groups. Results: Compared to women with normal weight, women with overweight or obesity before pregnancy had an increased risk of preterm birth; the RRs and 95\% Cls were 1.22 (95\% Cl: 1.08-1.37) and 1.30 (95\% Cl: 1.01-1.69), respectively. The greatest risk of extremely preterm birth was observed in obese women. The estimators were robust when considering the maternal age and rate of gestational weight gain (GWG) during pregnancy. Conclusions: Women with overweight and obesity had an increased risk of preterm birth regardless of GWG in early pregnancy. Our study suggests that it is beneficial to lose weight before conception for both overweight and obese women who plan to become pregnant.


Su et al.: Prepregnancy BMI and Preterm Birth

\section{Introduction}

Preterm birth is the second leading cause of death among children under 5 years of age worldwide. An estimated 15 million live-born babies are born prior to 37 weeks' gestation every year, with over 1.1 million born in China [1]. The incidence rate of preterm birth is anticipated to increase steeply over the next decade [2]. Multiple health indexes are associated with preterm birth, including childhood obesity [3], kidney diseases, and heart disease in adulthood [4-6]. The identification of modifiable factors to reduce preterm birth is urgently needed.

Although some causes of preterm birth have been identified, including multiple pregnancies and infectious and severe pregnancy complications, the etiology of two-thirds of preterm births is still unclear [7]. Previous studies have indicated that women with overweight or obesity have an increased risk of numerous negative pregnancy complications and outcomes, including still births [8], infant death [9], and a series of pregnancy complications [10-12], compared with women with normal weight; however, the association between weight and preterm birth is still inconsistent $[13,14]$. A systematic review, including studies in low- and middle-income countries, reported that maternal underweight rather than overweight or obesity is associated with an increased risk of preterm birth compared with maternal optimal weight [15]. A recent study based on the US National Vital Statistics System for 2016 and 2017 indicated that the association between obesity before pregnancy and preterm birth varied with age and ethnicity [16]. However, the body mass index (BMI) criteria for assessing the human weight range varied in previous studies, even among patients of the same ethnicity $[17,18]$, making the comparison of these studies difficult.

In the present study, we categorized the population into four groups according to the criteria for Asian women [19] and determined whether maternal overweight or obesity contributed to an increased risk of preterm birth in Chinese women.

\section{Materials and Methods}

This was a hospital-based retrospective cohort study. We used data from the electronic medical records system of Shanghai First Maternity and Infant Hospital, which covers almost one-third of the deliveries in Shanghai, China.

\section{Population}

We included all women who had a singleton live birth between January 2015 and December 2018 and complete data for prepregnancy weight, height, and gestational age at birth $(n=39,197)$. Women with implausible measurements of height $(<140 \mathrm{~cm})$, prepregnancy weight, or weight at initial prenatal care $(<35 \mathrm{~kg}, n=1,025)$, gestational age at birth ( $>44$ or $<22$ weeks, $n=37$ ), or chronic disease reports, including diabetes and hypertension $(n=1,539)$, were excluded. A total of 36,596 women were included in the final analysis.

\section{Exposure}

According to recommendations of appropriate BMI for Asian populations by the World Health Organization (WHO) [19], women were classified as underweight (BMI $<18.5 \mathrm{~kg} / \mathrm{m}^{2}$ ), normal weight (BMI 18.5 to $<23 \mathrm{~kg} / \mathrm{m}^{2}$ ), overweight (BMI 23 to $<27.5 \mathrm{~kg} / \mathrm{m}^{2}$ ), or obese (BMI $\geq 27.5 \mathrm{~kg} / \mathrm{m}^{2}$ ). 
Su et al.: Prepregnancy BMI and Preterm Birth

\section{Outcome}

The main outcome of interest was preterm birth, which was defined as delivery occurring before 37 weeks of gestation. Gestational age was calculated on the basis of the self-reported last menstrual date. We used the ultrasound-corrective gestational age for women with an irregular menstrual period. In the main analysis, we further subdivided preterm birth into three subtypes: extremely preterm birth ( $<28$ gestational weeks), very preterm birth (28-31 gestational weeks), and moderate preterm birth (32-36 gestational weeks). For comparisons with some other studies, we also used another classification of preterm birth in the sensitivity analysis, i.e., early preterm birth ( $<34$ gestational weeks) and late preterm birth (34-36 gestational weeks).

\section{Statistical Analysis}

The characteristics of the study participants were presented as means with standard deviations (SDs) for continuous variables and percentages for categorical variables. The differences in the characteristics among the four groups were also estimated by t tests for continuous variables and by $\chi^{2}$ tests for categorical variables.

Multivariate log-binomial regression models were used to estimate the relative risk (RR) and $95 \%$ confidence interval (95\% CI) for preterm birth and its subgroups in women with underweight, overweight, or obesity compared to women with normal weight. The following fixed set of covariates was defined a priori and included in the main models regardless of their difference in the different groups: maternal age $(<24$ years, 25-29 years, 30-34 years, 35+ years), paternal age ( $<24$ years, 25-29 years, 30-34 years, 35-39 years, $40+$ years), parity (nulliparous, multiparous), family history of diabetes and hypertension (yes, no), and mode of delivery (vaginal delivery, caesarean section).

To explore the effect modification of maternal age, we performed stratified analyses according to maternal age. In addition, we estimated the rate of gestational weight gain (GWG) in early pregnancy, which was calculated as weight at enrolment minus weight at prepregnancy and further divided by the weeks between the two measurements. As GWG is more likely to be a potential mediator for the association of maternal obesity and risk of preterm obesity, we performed stratified analyses according to the rate of GWG instead of adjusting it in the main models [20]. To limit potential inaccuracies in the prepregnancy $\mathrm{BMI}$, we refined the analyses for the rate of GWG in women who started prenatal care before 28 weeks $(n=22,313)$. As there are no standard categories for the rate of GWG in Chinese pregnant women, GWG was subdivided into three groups according to the quartiles (inadequate: [<25th percentile, P25], optimal: [P25-P75], excessive: [>P75]) in the study.

To assess the robustness of the findings, we rerun analyses by stratifying the women into (1) those without preterm birth before the current pregnancy; and (2) those without pregnancy complications, including gestational diabetes, gestational hypertension, preeclampsia, and placenta previa, which were potential mediators for the association of prepregnancy BMI and preterm birth. Considering the outliers might bias the estimators, we also performed analysis by excluding women with outlier value of weight ( $>80 \mathrm{~kg}$ ) or prepregnancy BMI $\left(>30 \mathrm{~kg} / \mathrm{m}^{2}\right)$ (i.e., the 99 th percentile). Finally, we also performed a similar analysis by categorizing preterm birth into early preterm birth and late preterm birth.

All analyses were conducted using the SAS 9.4 software package (SAS Institute Inc., Cary, NC, USA). 
Su et al.: Prepregnancy BMI and Preterm Birth

Table 1. Basic characteristics of the study population by prepregnancy BMI

\begin{tabular}{|c|c|c|c|c|c|}
\hline Characteristic & $\begin{array}{l}\text { Optimal weight } \\
(n=23,534)\end{array}$ & $\begin{array}{l}\text { Underweight } \\
(n=5,299)\end{array}$ & $\begin{array}{l}\text { Overweight } \\
(n=6,722)\end{array}$ & $\begin{array}{l}\text { Obesity } \\
(n=1,030)\end{array}$ & $\begin{array}{l}\text { Total } \\
(n=36,596)\end{array}$ \\
\hline Age, years & $29.7 \pm 3.7$ & $28.5 \pm 3.4$ & $30.6 \pm 4.0$ & $30.5 \pm 4.0$ & $29.7 \pm 3.8$ \\
\hline Spouse age, years & $30.8 \pm 4.4$ & $29.8 \pm 4.2$ & $31.7 \pm 4.7$ & $31.6 \pm 4.7$ & $30.8 \pm 4.5$ \\
\hline Gestational weeks & $38.9 \pm 1.3$ & $38.9 \pm 1.3$ & $38.8 \pm 1.4$ & $38.7 \pm 1.5$ & $38.9 \pm 1.4$ \\
\hline \multicolumn{6}{|l|}{ Parity, $n(\%)$} \\
\hline Nulliparous & $17,383(74)$ & $4,338(82)$ & $4,440(66)$ & $685(67)$ & $26,846(73)$ \\
\hline Multiparous & $6,162(26)$ & $961(18)$ & $2,282(34)$ & $345(33)$ & $9,750(27)$ \\
\hline \multicolumn{6}{|c|}{ History of preterm, $n(\%)$} \\
\hline No & 23,405 (99) & $5,266(99)$ & $6,659(99)$ & $1,021(99)$ & $36,351(99)$ \\
\hline Yes & $140(1)$ & $33(1)$ & $63(1)$ & $9(1)$ & $245(1)$ \\
\hline \multicolumn{6}{|l|}{ Mode of delivery, $n(\%)$} \\
\hline Vaginal delivery & $15,924(68)$ & $3,917(74)$ & $3,726(55)$ & $480(47)$ & $24,047(66)$ \\
\hline Caesarean section & $7,621(32)$ & $1,382(26)$ & $2,996(45)$ & $550(53)$ & $12,549(34)$ \\
\hline \multicolumn{6}{|c|}{ Pregnancy complications, $n(\%)$} \\
\hline No & $20,224(86)$ & $4,798(91)$ & $5,176(77)$ & $672(65)$ & $30,870(84)$ \\
\hline Yes & $3,321(14)$ & $501(9)$ & $1,546(23)$ & $358(35)$ & $5,726(16)$ \\
\hline \multicolumn{6}{|l|}{ Birth year } \\
\hline 2015 & $786(3)$ & $194(3)$ & $212(3)$ & $44(3)$ & $1,236(3)$ \\
\hline 2016 & $8,736(37)$ & $2,041(39)$ & $2,443(36)$ & $378(37)$ & $13,598(37)$ \\
\hline 2017 & $8,752(37)$ & $1,869(35)$ & $2,506(37)$ & $372(36)$ & $13,499(37)$ \\
\hline 2018 & $5,271(22)$ & $1,195(23)$ & $1,561(23)$ & $236(23)$ & $8,263(23)$ \\
\hline \multicolumn{6}{|c|}{ Gestational weeks at initial prenatal care, weeks, $n(\%)$} \\
\hline $1-12$ & $2,038(8)$ & $434(8)$ & $599(9)$ & $108(11)$ & $3,179(9)$ \\
\hline $13-20$ & $20,923(89)$ & $4,696(89)$ & $5,955(88)$ & $888(86)$ & $32,462(88)$ \\
\hline $21-27$ & $412(2)$ & $119(2)$ & $119(2)$ & $25(2)$ & $675(2)$ \\
\hline$>27$ & $172(1)$ & $50(1)$ & $49(1)$ & $9(1)$ & $280(1)$ \\
\hline
\end{tabular}

\section{Results}

There were 5,299 (14.5\%) women who were categorized as underweight, 23,545 (64.3\%) categorized as normal weight, 6,722 (18.4\%) categorized as overweight, and 1,030 (2.8\%) categorized as obese before pregnancy. The mean age of the study population was 29.7 (3.8) years. Basic characteristics of the study population according to the prepregnancy BMI were shown in Table 1. Compared with women with optimal weight before pregnancy, women with obesity were more likely to be multiparous (33 vs. $26 \%$ ), giving birth by caesarean section (53 vs. $32 \%$ ).

In the overall population, women with overweight (adjusted RR 1.22, 95\% CI: 1.08-1.38) or obesity (adjusted RR 1.30, 95\% CI: 1.00-1.69) had an overall increased risk of preterm birth (Table 2). When preterm birth was stratified into four subgroups according to gestational age, we observed an obviously increased risk for extremely preterm birth among women with obesity before pregnancy (adjusted RR 8.52, 95\% CI: 1.68-43.24).

When stratified according to the women's age, we observed that prepregnancy BMI was positively associated with an increased risk of preterm birth; most of the associations were nonsignificant due to the small sample sizes in each group. When the women were stratified into groups according to the rate of GWG, we observed that the effect size of obesity was increased regardless of the rate of GWG; however, all estimators were nonsignificant (Table $3)$.

We observed a similar association pattern when refining the analysis to women without previous preterm birth or without pregnancy complications. We performed a similar analysis 
Su et al.: Prepregnancy BMI and Preterm Birth

Table 2. Adjusted ${ }^{\mathrm{a}}$ relative risk ( $\left.95 \% \mathrm{CI}\right)$ for the associations between prepregnancy BMI and preterm birth and its subtypes

\begin{tabular}{lllll}
\hline Preterm birth & Underweight & Optimal weight & Overweight & Obese \\
\hline Overall & $1.05(0.90-1.22)$ & 1.00 (reference) & $1.22(1.08-1.38)$ & $1.30(1.02-1.69)$ \\
Moderately preterm birth & $1.06(0.91-1.24)$ & 1.00 (reference) & $1.22(1.08-1.38)$ & $1.23(0.94-1.61)$ \\
Very preterm birth & $0.21(0.03-1.57)$ & 1.00 (reference) & $0.74(0.28-1.98)$ & $2.67(0.79-9.08)$ \\
Extremely preterm birth & $1.66(0.33-8.32)$ & 1.00 (reference) & $2.77(0.83-9.15)$ & $8.26(1.63-41.88)$ \\
\hline
\end{tabular}

a Adjusted for maternal age, paternal age, parity (nulliparous, multiparous), family history of diabetes and hypertension (yes, no), and mode of delivery (vaginal delivery, caesarean section).

Table 3. Adjusted ${ }^{\text {a }}$ relative risk (95\% CI) for the associations between prepregnancy BMI and preterm birth by maternal age rate of gestational weight gain

\begin{tabular}{lcccc}
\hline & Underweight & Optimal weight & Overweight & Obese \\
\hline $\begin{array}{l}\text { Maternal age, years } \\
\quad<24\end{array}$ & $2.15(1.14-4.04)$ & 1.00 (reference) & $0.30(0.07-1.31)$ & $2.34(0.51-10.64)$ \\
$25-29$ & $0.98(0.78-1.21)$ & 1.00 (reference) & $1.16(0.94-1.42)$ & $1.34(0.86-2.07)$ \\
$30-34$ & $1.04(0.8-1.36)$ & 1.00 (reference) & $1.29(1.07-1.56)$ & $1.18(0.78-1.78)$ \\
$\quad \geq 35$ & $1.02(0.67-1.56)$ & 1.00 (reference) & $1.25(0.96-1.62)$ & $1.43(0.82-2.49)$ \\
Rate of gestational weight gain $\mathrm{b}$ & & & \\
Inadequate $(<$ P25) & $1.20(0.83-1.72)$ & 1.00 (reference) & $0.94(0.66-1.35)$ & $1.36(0.68-2.75)$ \\
Optimal (P25-P75) & $1.19(0.82-1.75)$ & 1.00 (reference) & $1.28(0.95-1.72)$ & $1.46(0.79-2.70)$ \\
Excessive ( $\geq$ P75) & $0.90(0.69-1.17)$ & 1.00 (reference) & $1.16(0.92-1.45)$ & $1.59(0.98-2.59)$ \\
\hline
\end{tabular}

${ }^{a}$ Adjusted for paternal age, parity, family history of diabetes and hypertension, mode of delivery, gestational period at initial prenatal care.

b Those women who started prenatal care after 28 weeks were excluded ( $n=14,283)$, and a total of 22,313 women were included in this analysis finally.

by categorizing preterm birth into early preterm birth and late preterm birth. We also observed that maternal obesity was associated with an increased risk for early preterm birth (data available upon request). After excluding women with outlier value of weight ( $>80 \mathrm{~kg}$ ) or prepregnancy BMI ( $>30 \mathrm{~kg} / \mathrm{m}^{2}$ ), the estimate also did not change obviously (data available upon request).

\section{Discussion/Conclusion}

In the retrospective cohort study, we observed that the obesity rate in Chinese women was relatively low (2.8\%), while nearly $20 \%$ of women were overweight. Our study indicated a significant increased risk of preterm birth among women with overweight or obesity before pregnancy. The association was obvious when focusing on extremely preterm birth.

Our study confirmed the results of two other studies performed in northern China that indicated that both overweight and obesity are associated with preterm birth [18]. A study performed in Qatar reported that approximately $16 \%$ of preterm births could be avoided if all mothers had a normal prepregnancy BMI according to the population attributable fractions, although they did not observe a significant association between obesity and risk of preterm birth among Arab women [21]. Our result regarding extremely preterm birth is also 
Su et al.: Prepregnancy BMI and Preterm Birth

consistent with results from a Swedish study [22]. A systematic review of studies from lowand middle-income countries reported that maternal underweight was significantly associated with an increased risk of preterm birth [15], and a hospital-based study from Lebanon also indicated an increased risk of preterm birth only among underweight women with low GWG [23]. The inconsistent results could be due to the different ethnicities. In addition, the definition of obesity was different among the studies, although both populations were Asian.

A previous study indicated that the effect of weekly GWG was significant during only the early period of pregnancy [24], while another study reported that both low and high rates of GWG in the second and third trimesters were positively associated with preterm birth [25]. To exclude potential confounding factors due to the rate of GWG, we performed a stratified analysis, and the results indicated that prepregnancy obesity was independently associated with an increased risk of preterm birth. It is worth noting that the results do not imply that weight gain control during pregnancy in women with optimal weight before pregnancy can be disregarded.

The biological mechanism of the effect of prepregnancy obesity on preterm obesity is still unclear. Moreover, little is known about how preterm birth occurs at a mechanistic level. From the labour onset viewpoint, the current understanding is that the change in the myometrium from a quiescent to a contractile state is accompanied by a shift from the activation of anti-inflammatory to pro-inflammatory signaling pathways, including the activation of chemokines (interleukin-8 [IL-8]), cytokines (IL-1 and -6), and contraction-associated proteins [26]. Additionally, some previous studies have reported that obesity is linked with the maternal cytokine profile during pregnancy. Polari et al. [27] reported that low-grade inflammation associated with obesity may not be alleviated by an increase in anti-inflammatory cytokines during pregnancy. Luciana et al. [28] also reported that maternal obesity creates a unique in utero environment, which could impair the placental transcriptome and have potential adverse consequences for placental structure and function, which might also highlight the mechanism of preterm birth.

The main strength of our study is the large sample size of Chinese women, which provides the ability to estimate the associations between prepregnancy obesity and subgroups of preterm births. In addition, as a tertiary maternal and infant hospital, our institute has the largest delivery burden in China, ensuring the representativeness of the study population. Our study also has some limitations. First, we did not have information on GWG by gestational week, as most of the values were not recorded in the electronic medical history in our institute. Second, similar to any other observational study, self-reported prepregnancy weight is imprecise, especially for women attending their initial clinic visiting in late pregnancy. However, the estimators were robust when the analysis was refined to those women who attended their first visit before 20 or 28 gestational weeks. Third, we do not have enough siblings with common mother to reduce potential confounding due to unobserved factors, such as genetic factors, smoking, and other individual characteristics as previous studies did $[29,30]$.

In conclusion, our study suggested that overweight or obesity before pregnancy is associated with an increased risk of preterm birth, independent of GWG in early pregnancy and maternal age.

\section{Acknowledgement}

We would like to express our appreciation to the Department of Obstetrics, Shanghai First Maternity and Infant Hospital, Tongji University School of Medicine, for supplying patient medical records. 
Su et al.: Prepregnancy BMI and Preterm Birth

\section{Statement of Ethics}

The study was approved by the Ethics Committee of Shanghai First Maternity and Infant Hospital, Tongji University School of Medicine. The study was performed in accordance with the approved guidelines.

\section{Disclosure Statement}

The authors have no conflicts of interest to declare.

\section{Funding Sources}

This work was supported by the National Natural Science Foundation of China (grant No. 81602860) the and Pudong Municipal Health Commission (grant No. PW2019D-9).

\section{Author Contributions}

Su wrote the manuscript and researched data. Huang, Li, and Du researched data, oversaw data collection and analysis, contributed to writing of the manuscript, and reviewed and edited the manuscript. All authors were involved in writing of the paper and had final approval of the submitted and published versions.

\section{References}

1 Vogel JP, Chawanpaiboon S, Watananirun K, Lumbiganon P, Petzold M, Moller AB, et al. Global, regional and national levels and trends of preterm birth rates for 1990 to 2014: protocol for development of World Health Organization estimates. Reprod Health. 2016;13(1):76.

2 Kramer H, Kalantar-Zadeh K. Obesity, preterm birth and kidney disease: a global epidemic. Nephrol Dial Transplant. 2019 Oct;34(10):1653-6.

3 Wood CT, Linthavong O, Perrin EM, Leviton A, Allred EN, Kuban KCK, et al. Antecedents of Obesity Among Children Born Extremely Preterm. Pediatrics. 2018 Nov;142(5). pii: e20180519.

4 Crump C, Friberg D, Li X, Sundquist J, Sundquist K. Preterm birth and risk of sleep-disordered breathing from childhood into mid-adulthood. Int J Epidemiol. 2019 Dec;48(6):2039-49.

5 Crump C, Howell EA, Stroustrup A, McLaughlin MA, Sundquist J, Sundquist K. Association of Preterm Birth With Risk of Ischemic Heart Disease in Adulthood. JAMA Pediatrics. 2019;173(8):736-43.

6 Crump C, Sundquist J, Winkleby MA, Sundquist K. Preterm birth and risk of chronic kidney disease from childhood into mid-adulthood: national cohort study. BMJ. 2019 May;365.11346 https://doi.org/10.1136/ bmj.11346.

7 Goldenberg RL, Culhane JF, Iams JD, Romero R. Epidemiology and causes of preterm birth. Lancet. 2008 Jan; 371(9606):75-84.

8 Flenady V, Koopmans L, Middleton P, Frøen JF, Smith GC, Gibbons K, et al. Major risk factors for stillbirth in high-income countries: a systematic review and meta-analysis. Lancet. 2011 Apr;377(9774):1331-40.

9 Chen A, Feresu SA, Fernandez C, Rogan WJ. Maternal obesity and the risk of infant death in the United States. Epidemiology. 2009;20(1):74-81.

10 Haddow JE, Metzger BE, Lambert-Messerlian G, Eklund E, Coustan D, Catalano P, et al. Maternal BMI, Peripheral Deiodinase Activity, and Plasma Glucose: Relationships Between White Women in the HAPO Study. J Clin Endocrinol Metab. 2019 Jul;104(7):2593-600.

11 Timpka S, Stuart JJ, Tanz LJ, Hu FB, Franks PW, Rich-Edwards JW. Postpregnancy BMI in the Progression From Hypertensive Disorders of Pregnancy to Type 2 Diabetes. Diabetes Care. 2019 Jan;42(1):44-9.

12 Knight-Agarwal CR, Williams LT, Davis D, Davey R, Cochrane T, Zhang H, et al. Association of BMI and interpregnancy BMI change with birth outcomes in an Australian obstetric population: a retrospective cohort study. BMJ Open. 2016 May;6(5):e010667. 
Su et al.: Prepregnancy BMI and Preterm Birth

13 Leung TY, Leung TN, Sahota DS, Chan OK, Chan LW, Fung TY, et al. Trends in maternal obesity and associated risks of adverse pregnancy outcomes in a population of Chinese women. BJOG. 2008 Nov;115(12):1529-37.

14 Huang A, Ji Z, Zhao W, Hu H, Yang Q, Chen D. Rate of gestational weight gain and preterm birth in relation to prepregnancy body mass indices and trimester: a follow-up study in China. Reprod Health. 2016;13(1):93.

15 Rahman MM, Abe SK, Kanda M, Narita S, Rahman MS, Bilano V, et al. Maternal body mass index and risk of birth and maternal health outcomes in low- and middle-income countries: a systematic review and meta-analysis. Obes Rev. 2015 Sep;16(9):758-70.

16 Liu B, Xu G, Sun Y, Du Y, Gao R, Snetselaar LG, et al. Association between maternal pre-pregnancy obesity and preterm birth according to maternal age and race or ethnicity: a population-based study. Lancet Diabetes Endocrinol. 2019;7(9):707-14.

17 Zhou Y, Li H, Zhang Y, Zhang L, Liu J, Liu J. Association of Maternal Obesity in Early Pregnancy with Adverse Pregnancy Outcomes: A Chinese Prospective Cohort Analysis. Obesity (Silver Spring). 2019 Jun;27 (6):1030-6.

18 Liu X, Du J, Wang G, Chen Z, Wang W, Xi Q. Effect of pre-pregnancy body mass index on adverse pregnancy outcome in north of China. Arch Gynecol Obstet. 2011 Jan;283(1):65-70.

19 Consultation WH; WHO Expert Consultation. Appropriate body-mass index for Asian populations and its implications for policy and intervention strategies. Lancet. 2004 Jan;363(9403):157-63.

20 Oken E, Kleinman KP, Belfort MB, Hammitt JK, Gillman MW. Associations of gestational weight gain with shortand longer-term maternal and child health outcomes. Am J Epidemiol. 2009 Jul;170(2):173-80.

21 Shaukat S, Nur U. Effect of prepregnancy maternal BMI on adverse pregnancy and neonatal outcomes: results from a retrospective cohort study of a multiethnic population in Qatar. BMJ Open. 2019 Sep;9(9):e029757.

22 Cnattingius S, Villamor E, Johansson S, Edstedt Bonamy AK, Persson M, Wikström AK, et al. Maternal obesity and risk of preterm delivery. JAMA. 2013 Jun;309(22):2362-70.

23 El Rafei R, Abbas HA, Charafeddine L, Nakad P, Al Bizri A, Hamod D, et al. Association of Pre-Pregnancy Body Mass Index and Gestational Weight Gain with Preterm Births and Fetal Size: an Observational Study from Lebanon. Paediatr Perinat Epidemiol. 2016 Jan;30(1):38-45.

24 Li C, Zeng L, Wang D, Dang S, Chen T, Watson V, et al. Effect of maternal pre-pregnancy BMI and weekly gestational weight gain on the development of infants. Nutr J. 2019 Jan;18(1):6.

25 Durie DE, Thornburg LL, Glantz JC. Effect of second-trimester and third-trimester rate of gestational weight gain on maternal and neonatal outcomes. Obstet Gynecol. 2011 Sep;118(3):569-75.

26 Romero R, Dey SK, Fisher SJ. Preterm labor: one syndrome, many causes. Science. 2014 Aug;345(6198):760-5.

27 Polari L, Kumar H, Rautava S, Salminen S, Isolauri E. Increase in serum Interleukin-10 does not alleviate proinflammatory MCP-1 production in obese pregnancies. Cytokine. 2018 Aug;108:67-70.

28 Lassance L, Haghiac M, Leahy P, Basu S, Minium J, Zhou J, et al. Identification of early transcriptome signatures in placenta exposed to insulin and obesity. Am J Obstet Gynecol. 2015 May;212(5):647 e1-11.

29 Ludwig DS, Currie J. The association between pregnancy weight gain and birthweight: a within-family comparison. Lancet. 2010 Sep;376(9745):984-90.

30 Averett SL, Fletcher EK. Prepregnancy Obesity and Birth Outcomes. Matern Child Health J. 2016 Mar;20(3): 655-64. 